\title{
O fonema /r/ e as alterações do sistema estomatognático, idade, gênero e gravidade no desvio fonológico
}

\section{The phoneme /r/ and stomatognathic system alterations, age, gender and severity level in phonological disorders}

\author{
Caroline Marini ${ }^{1}$, Ana Rita Brancalioni' ${ }^{1}$, Marileda Barichello Gubiani ${ }^{1}$, Giséli Pereira de Freitas ${ }^{1}$, \\ Márcia Keske-Soares ${ }^{2}$ Cláudio Cechella²
}

\begin{abstract}
RESUMO
Objetivo: Comparar a produção do fonema/r/ em crianças com desvio fonológico (DF), e as alterações no sistema estomatognático (SEG) considerando as variáveis de idade, gênero e gravidade do DF. Métodos: A amostra constituiu-se de 113 crianças com DF e alterações do SEG, de ambos os gêneros, e idades entre 4 anos e 8 anos e 11 meses, divididos em dois grupos, considerando a faixa etária: pré-escolar (4 anos a 5 anos e 11 meses) e escolar (6 anos a 8 anos e 11 meses). Realizou-se Avaliação Fonológica da Criança e avaliação do SEG. A amostra foi dividida, quanto à gravidade do DF, em dois grupos, Graus mais Graves (GG) e Graus mais Leves (GL). Quanto ao sistema fonológico (SF), a mesma foi dividida em GI: /r/ estabelecido; e GII: /r/ não estabelecido. Os dados coletados foram tabulados, analisados e, posteriormente, submetidos a tratamento estatístico. Resultados: Não foi observada diferença na postura, tonicidade e mobilidade de língua, lábios e bochechas, bem como nas estruturas e funções do SEG entre GI e GII. O GI obteve melhor desempenho apenas na vibração de língua. Verificou-se relação entre a aquisição do fonema /r/ e o aumento da idade, não havendo correlação com o gênero. Ainda, os GG de DF são mais frequentes no GII, e os GL no GI. Conclusão: Não há relação entre as alterações do SF e das estruturas e funções do SEG em crianças com DF, havendo relação deste apenas com a idade das mesmas.
\end{abstract}

Descritores: Sistema estomatognático; Fala; Criança; Distúrbios da fala; Transtornos da articulação

\section{INTRODUÇÃO}

A prevalência, na clínica fonoaudiológica, de crianças com desvios na produção de determinados fonemas é significativa, tanto em idade de aquisição fonológica, quanto em idades posteriores à aquisição ${ }^{(1)}$. Nestes casos, a dificuldade nesta aquisição em período adequado caracteriza o desvio fonológico (DF) ${ }^{(2)}$. Este afeta apenas o nível fonológico, da organização linguística ${ }^{(3)}$.

Alguns autores ${ }^{(4-6)}$ referem que a aquisição e o desenvolvimento da fala dependem tanto do desenvolvimento dos órgãos e funções do sistema estomatognático (SEG) quanto da organização do SF, ou seja, de aspectos fonéticos e fonológi$\cos$. Outros ${ }^{(7,8)}$, igualmente, não descartam a possibilidade de

Trabalho realizado no Curso de Fonoaudiologia, Universidade Federal de Santa Maria - UFSM - Santa Maria (RS), Brasil.

(1) Programa de Pós-graduação (Mestrado) em Distúrbios da Comunicação Humana, Universidade Federal de Santa Maria - UFSM - Santa Maria (RS), Brasil.

(2) Departamento de Fonoaudiologia, Universidade Federal de Santa Maria - UFSM - Santa Maria (RS), Brasil.

Endereço para correspondência: Márcia Keske-Soares. RST 287 (Faixa Nova de Camobi), 900, Cerrito, Santa Maria (RS), Brasil, CEP: 97060-500.

E-mail: keske-soares@uol.com.br

Recebido em: 21/8/2009; Aceito em: 6/12/2010 imprecisão articulatória, além das dificuldades apresentadas com a organização dos sons da fala.

A presença de alterações de fala em idade pré-escolar pode advir de problemas do SEG e/ou da organização dos fonemas, pois a fala requer coordenação complexa e planejamento de movimentos de lábios e de língua, que resultam na produção dos sons. Quando há alteração quanto à tonicidade e praxias, pode haver interferência, também, na produção dos fonemas ${ }^{(6,9,10)}$. Uma pesquisa ${ }^{(11)}$ afirma haver estreita relação entre a fala e a produção de gestos, e forte correlação entre fala/linguagem e praxias.

A aquisição fonológica estabelece estreita relação com o desenvolvimento do controle motor da fala, pois, para produzir uma sequência de sons, a criança necessita de habilidade para acionar o trato vocal e ordenar os movimentos dos músculos em sequência $^{(12,13)}$. Há, dessa forma, uma ligação neurofisiológica comum entre o planejamento da fala e a motricidade fina, que se encontram alterados em crianças com $\mathrm{DF}^{(2)}$. O comprometimento da fala é verificado apenas quando há significativas anormalidades miofuncionais e não por mínimas imprecisões, uma vez que o mecanismo da fala é altamente flexível e adaptável ${ }^{(14)}$.

Estudos ${ }^{(15,16)}$ referem que os movimentos rápidos, sincrônicos e precisos da língua são fundamentais para a articulação adequada, principalmente do rótico alveolar simples, o /r/. 
A dificuldade de categorização fonológica, apontada como a causa mais provável para essas dificuldades, não pode ser considerada como o único fator causador das mesmas, sendo necessário considerar, ainda, os fatores fonético-articulatórios ${ }^{(7)}$. Por isso, avaliar o SEG é imprescindível para um adequado diagnóstico dos distúrbios da fala ${ }^{(10)}$.

As crianças tentam primeiramente produzir palavras que contenham sons que estejam dentro de suas capacidades articulatórias ${ }^{(17)}$. Os inventários iniciais das crianças contem, em geral, oclusivas e nasais, aparecendo, posteriormente, as fricativas e as líquidas. A classe das líquidas é a de maior alteração e dificuldade de produção no Português Brasileiro - $\mathrm{PB}^{(18)}$.

Quanto à aquisição dos róticos, que são assim denominados por suas similaridades acústicas e padrão fonológico comuns às líquidas laterais, no PB há foneticamente dois róticos: um em posição de onset simples, ou seja, o tap (' $r$ ' fraco); e a fricativa velar (' $r$ ' forte) ${ }^{(19)}$. A pronúncia dos róticos é bastante variada, tanto entre as diferentes línguas quanto dentro de uma mesma língua ${ }^{(20)}$. Portanto, tanto aspectos fonológicos como fonéticos, como as habilidades motoras linguais, estão envolvidos na aquisição do fonema $/ \mathrm{r}^{(7,21)}$.

Há indicação de que a praxia de vibrar a língua só é executada de forma adequada após a aquisição do fonema $/ \mathrm{r} /$ no SF da criança ${ }^{(21)}$. É comum ocorrer distorção na produção do /r/, sendo este o fonema mais susceptível a apresentar distorção na classe das líquidas ${ }^{(22,23)}$.

A produção da fala e as alterações do SEG estão diretamente relacionadas à estimulabilidade ${ }^{(21)}$, que é uma medida de produção importante para o reconhecimento das dificuldades de fala, tanto em nível fonético, fonológico ou de percepção ${ }^{(24)}$.

Evidencia-se que há escassez de estudos relacionando a produção da líquida não-lateral /r/ e os resultados da avaliação do SEG, considerando-se sua influência na produção correta deste fonema, que é o mais frequentemente acometido no DF, o que favorece um adequado diagnóstico e tratamento do DF. Assim, este estudo tem como objetivo comparar a produção do fonema /r/ em relação às alterações no SEG, idade, gênero e gravidade do DF.

\section{MÉTODOS}

Esta pesquisa foi realizada a partir do banco de dados de projetos devidamente aprovados e registrados no Comitê de Ética em Pesquisa (CEP) da Universidade Federal de Santa Maria (UFSM) sob números 046/02 e 108/05.

A participação das crianças foi autorizada, mediante a assinatura do Termo de Consentimento Livre e Esclarecido pelos pais e/ou responsáveis.

A amostra constituiu-se de 113 crianças de ambos os gêneros, sendo 72 do gênero masculino e 41 do feminino, com idades variando entre 4 anos e 8 anos e 11 meses, sendo divididos em dois grupos por faixa etária: idade pré-escolar (4 a 5 anos e 11 meses) composto por 52 crianças, e idade escolar (6 a 8 anos e 11 meses) com 61 crianças.

As crianças participantes apresentavam diagnóstico de DF, estabelecido a partir das avaliações realizadas previamente nos projetos de pesquisa. Para este estudo somente foram considerados os dados referentes às avaliações fonológica e do SEG.
Foram também submetidas às avaliações otorrinolaringológica e audiológica. Todos os resultados coletados faziam parte do período pré-tratamento.

A avaliação do SF foi realizada por meio da aplicação da Avaliação Fonológica da Criança (AFC) ${ }^{(25)}$, estando os dados transcritos apresentados com análise contrastiva. A partir dos resultados desta análise determinou-se a gravidade do DF, através do cálculo do Percentual de Consoantes Corretas-Revisado (PCC-R) ${ }^{(26)}$, sendo este classificado como: Desvio Leve (DL) - percentuais entre 86 e 100\%; Moderado-Leve (DML) - percentuais entre 66 e 85\%; Moderado-Grave (DMG) - percentuais variando entre 51 a $65 \%$; e Grave (DG) - percentuais menores que $50 \%$.

As crianças foram divididas em dois grupos, de acordo com a gravidade do DF: Graus mais Graves (GG), nos quais foram incluídas as com DG e DMG (n=18); e Graus mais Leves (GL) incluídas as com DL e DML (n=95).

Quanto ao SF, a amostra foi dividida em dois grupos: GI: /r/ estabelecido - composto por 39 crianças que apresentavam o $/ \mathrm{r} /$ estabelecido no SF, em posição de onset simples; e GII: /r/ não estabelecido - composto por 74 crianças com o /r/ não estabelecido no SF, na mesma posição de sílaba. O critério utilizado para considerar o fonema estabelecido no SF foi o de produção correta igual ou superior a $80 \%$, sendo que para percentuais inferiores, o fonema foi considerado não estabelecido ${ }^{(27)}$.

A avaliação do SEG foi realizada por meio do protocolo desenvolvido na UFSM e baseado em avaliações já existentes ${ }^{(28)}$. Nesta avaliação foram verificados aspectos referentes à sensibilidade (intra e extra-oral, além de possível presença de reflexos patológicos), propriocepção, morfologia, postura, tônus e mobilidade dos órgãos fonoarticulatórios (lábios, língua, bochechas, palato duro e mole, arcada dentária e mandíbula). Além disso, foram avaliadas as funções de sucção, mastigação, deglutição e respiração, por meio de tarefas que faziam parte, igualmente, do protocolo citado anteriormente. Neste, solicitou-se às crianças que bebessem água com canudo e, após, diretamente em um copo. A forma como a criança deglutia e sugava era observada pela examinadora. Da mesma forma, observou-se a mastigação, através da ingestão de bolachas e observação da forma como esta ocorria.

A fim de verificar a associação entre a aquisição do fonema /r/ e a postura, tonicidade e mobilidade da língua; postura, tonicidade e mobilidade de lábios e postura, tonicidade e mobilidade de bochechas foi utilizado o teste Qui-quadrado, complementado pela Análise dos Resíduos Ajustados. Para verificar a relação entre a aquisição do fonema /r/ e a mobilidade, postura e tonicidade dos lábios, língua, bochechas, e do tipo de oclusão dental, utilizou-se o teste de Correlação de Pearson. Por fim, com o objetivo de verificar associação entre a aquisição do fonema $/ \mathrm{r} /$ e as variáveis gênero, idade e gravidade do DF utilizou-se o teste Qui-quadrado. Os testes estatísticos foram realizados no software Stata 10.0 e o nível de significância adotado foi de $\mathrm{p}<0,05$.

\section{RESULTADOS}

A Tabela 1 compara postura, tonicidade e mobilidade da língua entre GI e GII. Observa-se que, para as variáveis postura e tonicidade, não houve diferença entre os grupos. 
Quanto à mobilidade, verifica-se que as ações de protruir, retrair, lateralizar (interna e externamente), estalar, elevar, abaixar, alargar e afinar a língua foram realizadas pela maioria das crianças, independente do fonema /r/ estar ou não estabelecido no seu SF. Ainda, não houve diferença entre os grupos para nenhuma dessas variáveis pesquisadas.

Além disso, observa-se que houve diferença significativa para a ação de vibrar a língua. A Análise dos Resíduos Ajustados indica que houve associação positiva para o GI realizar vibração de língua e associação negativa para GII na tarefa de vibração de língua.

Na Tabela 2 compara-se postura, tonicidade e mobilidade dos lábios entre GI e GII. Quanto à postura e à tonicidade não houve diferença para o fonema $/ \mathrm{r} /$ estar ou não estabelecido no $\mathrm{SF}$ das crianças. No que diz respeito à mobilidade, verifica-se que as ações de protruir, estirar, lateralizar, contrair, soprar e vibrar os lábios foram realizadas pela maioria das crianças de ambos os grupos. Não houve diferença para nenhuma dessas variáveis. Verifica-se também que a maioria das crianças, independente do fonema $/ \mathrm{r} /$ estar ou não estabelecido, apresentou maior dificuldade na praxia de assobiar.

A Tabela 3 compara postura, tonicidade e mobilidade de bochechas entre as crianças do GI e do GII. Observa-se que para as variáveis postura e tonicidade não houve diferença entre os grupos. Quanto à mobilidade, as ações de inflar as bochechas separadamente ou juntas foram realizadas pela maioria das crianças, independente do fonema /r/ estar ou não estabelecido no SF das mesmas.

A Tabela 4, por sua vez, revela a correlação entre a aquisição do fonema/r/ e a mobilidade dos órgãos do SEG. A variável que apresentou maior coeficiente de correlação com o fonema /r/ estar ou não estabelecido no SF, foi a vibração de língua, que apresenta uma correlação forte e positiva.

Além disso, os valores dos coeficientes de correlação próximos a zero, para as demais variáveis estudadas, mostram não haver relação entre estas. Ou seja, as variáveis pesquisadas

Tabela 1. Comparação da aquisição do fonema /r/ para postura, tonicidade e mobilidade da língua

\begin{tabular}{|c|c|c|c|c|c|c|c|}
\hline \multirow{3}{*}{ Língua } & & & \multicolumn{4}{|c|}{ Aquisição do fonema /r/ } & \multirow{3}{*}{ Valor de $\mathrm{p}$} \\
\hline & & & \multicolumn{2}{|c|}{ Gl } & \multicolumn{2}{|c|}{ Gll } & \\
\hline & & & $\mathrm{n}$ & $\%$ & $\mathrm{n}$ & $\%$ & \\
\hline \multirow{3}{*}{ Postura } & \multicolumn{2}{|c|}{ Contra os dentes incisivos superiores } & 16 & 41,03 & 24 & 32,43 & 0,614 \\
\hline & \multicolumn{2}{|c|}{ Contra os dentes incisivos inferiores } & 15 & 38,46 & 35 & 47,30 & \\
\hline & \multicolumn{2}{|l|}{ Entre os dentes } & 8 & 20,51 & 15 & 20,27 & \\
\hline \multirow{3}{*}{ Tonicidade } & \multicolumn{2}{|l|}{ Normotenso } & 37 & 94.87 & 68 & 91,89 & 0,582 \\
\hline & \multicolumn{2}{|l|}{ Tônus aumentado } & 0 & 0,00 & 2 & 2,70 & \\
\hline & \multicolumn{2}{|l|}{ Tônus reduzido } & 2 & 5,13 & 4 & 5,41 & \\
\hline \multirow{20}{*}{ Mobilidade } & \multirow{2}{*}{ Protruir } & Realiza & 38 & 97,44 & 71 & 95,95 & 0,571 \\
\hline & & Não realiza & 1 & 2,56 & 3 & 4,05 & \\
\hline & \multirow{2}{*}{ Retrair } & Realiza & 37 & 94,87 & 72 & 97,30 & 0,429 \\
\hline & & Não realiza & 2 & 5,13 & 2 & 2,70 & \\
\hline & \multirow{2}{*}{ Lateralizar internamente } & Realiza & 35 & 89,74 & 69 & 93,24 & 0,376 \\
\hline & & Não realiza & 4 & 10,26 & 5 & 6,76 & \\
\hline & \multirow{2}{*}{ Lateralizar externamente } & Realiza & 36 & 92,31 & 69 & 93,24 & 0,565 \\
\hline & & Não realiza & 3 & 7,69 & 5 & 6,76 & \\
\hline & \multirow{2}{*}{ Estalar } & Realiza & 31 & 79,49 & 61 & 82,43 & 0,443 \\
\hline & & Não realiza & 8 & 20,51 & 13 & 17,57 & \\
\hline & \multirow{2}{*}{ Vibrar } & Realiza & $13^{(+)}$ & 33,33 & $9^{(-)}$ & 12,16 & $0,014^{*}$ \\
\hline & & Não realiza & $26^{(-)}$ & 66,67 & $65^{(+)}$ & 87,84 & \\
\hline & \multirow{2}{*}{ Elevar a ponta } & Realiza & 34 & 87,18 & 56 & 75,68 & 0,114 \\
\hline & & Não realiza & 5 & 12,82 & 18 & 24,32 & \\
\hline & \multirow{2}{*}{ Abaixar a ponta } & Realiza & 37 & 94,87 & 65 & 87,84 & 0,196 \\
\hline & & Não realiza & 2 & 5,13 & 9 & 12,16 & \\
\hline & \multirow{2}{*}{ Alargar } & Realiza & 32 & 82,05 & 69 & 93,36 & 0,068 \\
\hline & & Não realiza & 7 & 17,95 & 5 & 6,76 & \\
\hline & \multirow{2}{*}{ Afilar } & Realiza & 29 & 74,36 & 43 & 58,11 & 0,1330 \\
\hline & & Não realiza & 10 & 25,64 & 31 & 41,89 & \\
\hline
\end{tabular}

*Valores significativos ( $p<0,05)$ - Teste Qui-quadrado

Análise de resíduos: (+) associação significativa positiva (-) associação significativa negativa

Legenda: $\mathrm{GI}$ = grupo I - fonema /r/ não estabelecido; GII = grupo II - fonema / $\mathrm{r} /$ estabelecido 
Tabela 2. Comparação da aquisição do fonema /r/ para postura, tonicidade e mobilidade dos lábios

\begin{tabular}{|c|c|c|c|c|c|c|c|}
\hline \multirow{3}{*}{ Lábios } & & & \multicolumn{4}{|c|}{ Aquisição do fonema /r/ } & \multirow{3}{*}{ Valor de $p$} \\
\hline & & & \multicolumn{2}{|c|}{ GI } & \multicolumn{2}{|c|}{ GII } & \\
\hline & & & $n$ & $\%$ & $\mathrm{n}$ & $\%$ & \\
\hline \multirow{2}{*}{ Postura } & \multicolumn{2}{|l|}{ Unidos } & 28 & 71,79 & 59 & 79,73 & 0,235 \\
\hline & \multicolumn{2}{|l|}{ Separados } & 11 & 28,21 & 15 & 20,27 & \\
\hline \multirow{3}{*}{ Tonicidade } & \multicolumn{2}{|l|}{ Normotenso } & 30 & 76,92 & 57 & 77,03 & 0,656 \\
\hline & \multicolumn{2}{|l|}{ Tônus aumentado } & 3 & 7,69 & 9 & 12,16 & \\
\hline & \multicolumn{2}{|l|}{ Tônus reduzido } & 6 & 15,38 & 8 & 10,81 & \\
\hline \multirow{14}{*}{ Mobilidade } & \multirow{2}{*}{ Protruir } & Realiza & 38 & 97,44 & 72 & 97,30 & 0,727 \\
\hline & & Não realiza & 1 & 2,56 & 2 & 2,70 & \\
\hline & \multirow{2}{*}{ Estirar } & Realiza & 35 & 89,74 & 70 & 94,59 & 0,277 \\
\hline & & Não realiza & 4 & 10,26 & 4 & 5,41 & \\
\hline & \multirow{2}{*}{ Lateralizar } & Realiza & 22 & 56,41 & 47 & 63,53 & 0,296 \\
\hline & & Não realiza & 17 & 43,51 & 27 & 36,49 & \\
\hline & \multirow{2}{*}{ Contrair } & Realiza & 36 & 92,31 & 66 & 89,19 & 0,433 \\
\hline & & Não realiza & 3 & 7,69 & 8 & 10,81 & \\
\hline & \multirow{2}{*}{ Vibrar } & Realiza & 24 & 61,54 & 28 & 51,35 & 0,202 \\
\hline & & Não realiza & 15 & 38,46 & 36 & 48,65 & \\
\hline & \multirow{2}{*}{ Soprar } & Realiza & 38 & 97,44 & 72 & 97,30 & 0,727 \\
\hline & & Não realiza & 1 & 2,56 & 2 & 2,70 & \\
\hline & \multirow{2}{*}{ Assobiar } & Realiza & 18 & 46,15 & 25 & 33,78 & 0,139 \\
\hline & & Não realiza & 21 & 53,85 & 49 & 66,22 & \\
\hline
\end{tabular}

Teste Qui-quadrado $(p<0,05)$

Legenda: $\mathrm{GI}$ = Grupo I fonema /r/ não estabelecido; GII = Grupo II fonema $/ \mathrm{r} /$ estabelecido

Tabela 3. Comparação da aquisição do fonema /r/ para postura, tonicidade e mobilidade das bochechas

\begin{tabular}{|c|c|c|c|c|c|c|c|}
\hline \multirow{3}{*}{ Bochechas } & & & \multicolumn{4}{|c|}{ Aquisição do fonema /r/ } & \multirow{3}{*}{ Valor de $p$} \\
\hline & & & \multicolumn{2}{|c|}{ GI } & \multicolumn{2}{|c|}{ GII } & \\
\hline & & & $\mathrm{n}$ & $\%$ & $\mathrm{n}$ & $\%$ & \\
\hline \multirow{2}{*}{ Postura } & \multicolumn{2}{|l|}{ Simétrico } & 38 & 97,44 & 69 & 93,24 & 0,322 \\
\hline & \multicolumn{2}{|l|}{ Assimétrico } & 1 & 2,56 & 5 & 6,76 & \\
\hline \multirow{3}{*}{ Tonicidade } & \multicolumn{2}{|l|}{ Normotenso } & 33 & 84,62 & 57 & 77,03 & 0,655 \\
\hline & \multicolumn{2}{|l|}{ Tônus aumentado } & 1 & 2,56 & 3 & 4,05 & \\
\hline & \multicolumn{2}{|l|}{ Tônus reduzido } & 5 & 12,82 & 14 & 18,92 & \\
\hline \multirow{6}{*}{ Mobilidade } & \multirow{2}{*}{ Inflar a direita } & Realiza & 28 & 71,79 & 53 & 71,62 & 0,583 \\
\hline & & Não realiza & 11 & 28,21 & 21 & 28,38 & \\
\hline & \multirow{2}{*}{ Inflar a esquerda } & Realiza & 30 & 76,92 & 54 & 72,97 & 0,413 \\
\hline & & Não realiza & 9 & 23,08 & 20 & 27,03 & \\
\hline & \multirow{2}{*}{ Inflar ambas } & Realiza & 39 & 100,00 & 74 & 100,00 & * \\
\hline & & Não realiza & 0 & 0,00 & 0 & 0,00 & \\
\hline
\end{tabular}

Teste Qui-quadrado $(p<0,05)$

Legenda: $\mathrm{GI}$ = Grupo I fonema /r/ não estabelecido; GII = Grupo II fonema /r/ estabelecido; * = não representativo para análise estatística

parecem não exercer influência sobre o fato de a criança ter ou não o fonema /r/ estabelecido em seu SF.

Na Tabela 5 é apresentada a correlação entre aquisição do fonema /r/ e postura e tonicidade de lábios, língua e bochechas. Os valores dos coeficientes de correlação próximos a zero mostram não haver relação, isto é, as variáveis pesquisadas parecem não influenciar a aquisição do fonema $/ \mathrm{r} /$.

A Figura 1 compara a aquisição do fonema /r/ com o tipo de oclusão dental. Observa-se que em ambos os grupos os maiores percentuais referem-se à oclusão normal, sendo esta diferença significativa $(\mathrm{p}=0,0071)$. Além disso, mordida profunda e cruzada tiveram ocorrência inferior a 5\%. Ainda, a análise estatística permite inferir que a oclusão normal é a mais frequentemente encontrada em ambos os grupos.

A Figura 2 apresenta a comparação entre a aquisição do fonema /r/ e as funções do SEG. A análise estatística com o 
Tabela 4. Correlação entre aquisição do fonema /r/ e mobilidade dos órgãos do SE

\begin{tabular}{|c|c|c|c|}
\hline \multirow[b]{2}{*}{ Órgão } & \multirow[b]{2}{*}{ Ação } & \multicolumn{2}{|c|}{ Aquisição do fonema /r/ } \\
\hline & & $\begin{array}{c}\text { Coeficiente de } \\
\text { correlação }\end{array}$ & Valor de $p$ \\
\hline \multirow{7}{*}{ Lábios } & Protruir & 0,0439 & $<0,050$ \\
\hline & Estirar & 0,1879 & $<0,050$ \\
\hline & Lateralizar & 0,0682 & $<0,050$ \\
\hline & Contrair & 0,1297 & $<0,050$ \\
\hline & Vibrar & 0,2672 & $<0,050$ \\
\hline & Soprar & 0,0439 & $<0,050$ \\
\hline & Assobiar & 0,3563 & $<0,050$ \\
\hline \multirow{9}{*}{ Língua } & Protruir & 0,1042 & $<0,050$ \\
\hline & Retrair & 0,0778 & $<0,050$ \\
\hline & Vibrar & 0,8601 & $<0,050$ \\
\hline & Lateralizar interna & 0,0751 & $<0,050$ \\
\hline & Lateralizar externa & 0,0115 & $<0,050$ \\
\hline & Elevar a ponta & 0,4162 & $<0,050$ \\
\hline & Abaixar a ponta & 0,3239 & $<0,050$ \\
\hline & Alargar & 0,0763 & $<0,050$ \\
\hline & Afinar & 0,5219 & $<0,050$ \\
\hline \multirow{3}{*}{ Bochechas } & Inflar a direita & 0,0404 & $<0,050$ \\
\hline & Inflar a esquerda & 0,1140 & $<0,050$ \\
\hline & Inflar ambas & * & * \\
\hline \multirow{2}{*}{ Mandíbula } & Abrir/fechar & * & * \\
\hline & Lateralizar & * & * \\
\hline
\end{tabular}

Teste de Correlação de Pearson $(p<0,05)$

Legenda: $\mathrm{GI}$ = grupo I - fonema /r/ não estabelecido; GII = grupo II - fonema $/ r /$ estabelecido; ${ }^{*}$ = não representativo para análise estatística

Tabela 5. Correlação entre aquisição do fonema /r/ (estar postura e tonicidade de lábios, língua e bochechas

\begin{tabular}{llcc}
\hline Órgãos & \multicolumn{2}{c}{ Aquisição do fonema /r/ } \\
\cline { 3 - 4 } & & $\begin{array}{c}\text { Coeficiente de } \\
\text { Correlação }\end{array}$ & Valor de $p$ \\
\hline Lábios & Postura & $-0,0896$ & $<0,050$ \\
& Tonicidade & $-0,0322$ & $<0,050$ \\
Língua & Postura & $-0,0012$ & $<0,050$ \\
& Tonicidade & 0,0334 & $<0,050$ \\
Bochechas & Postura & 0,0889 & $<0,050$ \\
& Tonicidade & 0,0862 & $<0,050$ \\
\hline
\end{tabular}

Teste de Correlação de Pearson $(p<0,05)$

Legenda: $\mathrm{Gl}$ = grupo I - fonema /r/ não estabelecido; GII = grupo II - fonema $/ \mathrm{r} /$ estabelecido

teste Qui-quadrado revelou não haver diferença entre os grupos para sucção, deglutição, mastigação e respiração.

A Figura 3 ilustra a aquisição do fonema /r/ conforme a idade. Verifica-se que a maioria das crianças do GI encontra-se em idade escolar, enquanto que no GII a maioria das crianças está em idade pré-escolar. Assim, o percentual de crianças com o fonema $/ r$ / estabelecido em seu SF cresce com o aumento da idade. A análise estatística com o teste Qui-quadrado identifi-

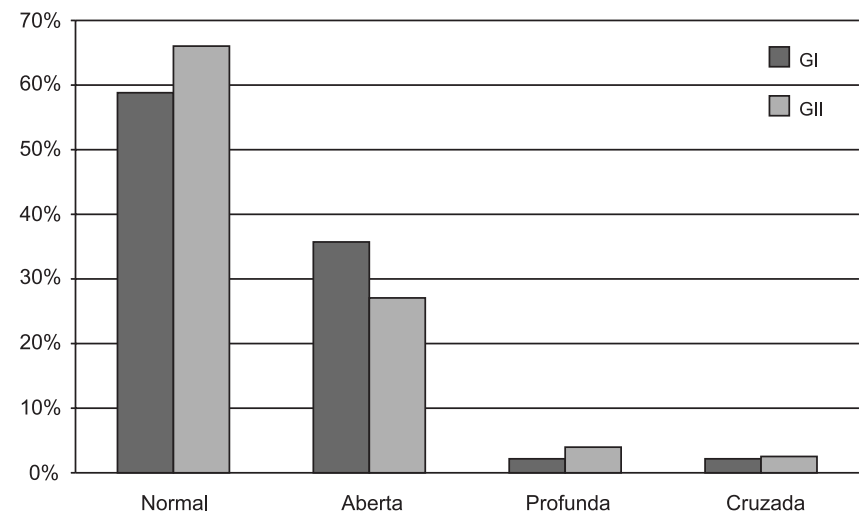

Legenda: $\mathrm{GI}$ = grupo I - fonema /r/ não estabelecido; GII = grupo II - fonema /r/ estabelecido

Figura 1. Aquisição do fonema /r/ segundo o tipo de oclusão dental

cou diferença entre os grupos $(\mathrm{p}=0,0490)$.

A Figura 4 apresenta a aquisição do fonema /r/ segundo a variável gênero. Observa-se que em ambos os grupos o percentual é maior para o gênero masculino do que para o feminino. Contudo, esta diferença não é significativa $(\mathrm{p}=0,1670)$.

$\mathrm{Na}$ Figura 5 é ilustrada a aquisição do fonema/r/ segundo a gravidade do DF. Verifica-se que GG de DF são mais frequentes no GII, enquanto que GL estão mais presentes no GI, sendo esta diferença significativa $(\mathrm{p}=0,0030)$.

\section{DISCUSSÃO}

Estudos $^{(6,11)}$ afirmam que a tonicidade alterada de órgãos fonoarticulatórios pode interferir na produção dos fonemas, sendo identificada como frequente a alteração de tônus muscular nos órgãos do SEG em crianças com queixa de fala ${ }^{(9)}$. No presente estudo não se verificou relação entre a tonicidade e a produção do fonema /r/. Esse resultado pode ser justificado pelo fato de as crianças deste estudo apresentarem diagnóstico de DF apenas, sem características fonéticas evidentes (apenas com alterações no SEG).

Quanto à mobilidade da língua, as praxias de protruir, retrair, lateralizar, elevar e estalar a língua foram realizadas pela maioria das crianças, independente de apresentarem ou não dificuldade na produção do fonema /r/. Além disso, o GII apresentou menor percentual de realização da praxia de afilar a língua, quando comparado com o GI, sendo que tais achados estão de acordo com a literatura ${ }^{(15,16)}$.

Os resultados relacionados à praxia de alargar a língua mostraram que as crianças da presente pesquisa não apresentaram dificuldade na realização de tal praxia. Esse achado difere de outro estudo $^{(16)}$, que refere que crianças com dificuldade na produção do /r/ também apresentavam dificuldade na praxia de alargar a língua. Indica também que crianças que não produzem $\mathrm{o} / \mathrm{r} /$ apresentam dificuldade em dissociar as praxias de afilar e alargar a língua, já que na produção do /r/ parte da língua encontra-se alargada, e outra, afilada.

Cabe ressaltar que a praxia de vibrar a língua foi a única que mostrou diferença significativa na presente pesquisa. $\mathrm{O}$ fato de vibrar a língua não ter sido realizado pela maioria das crianças, de ambos os grupos, sugere que esta é a praxia mais 

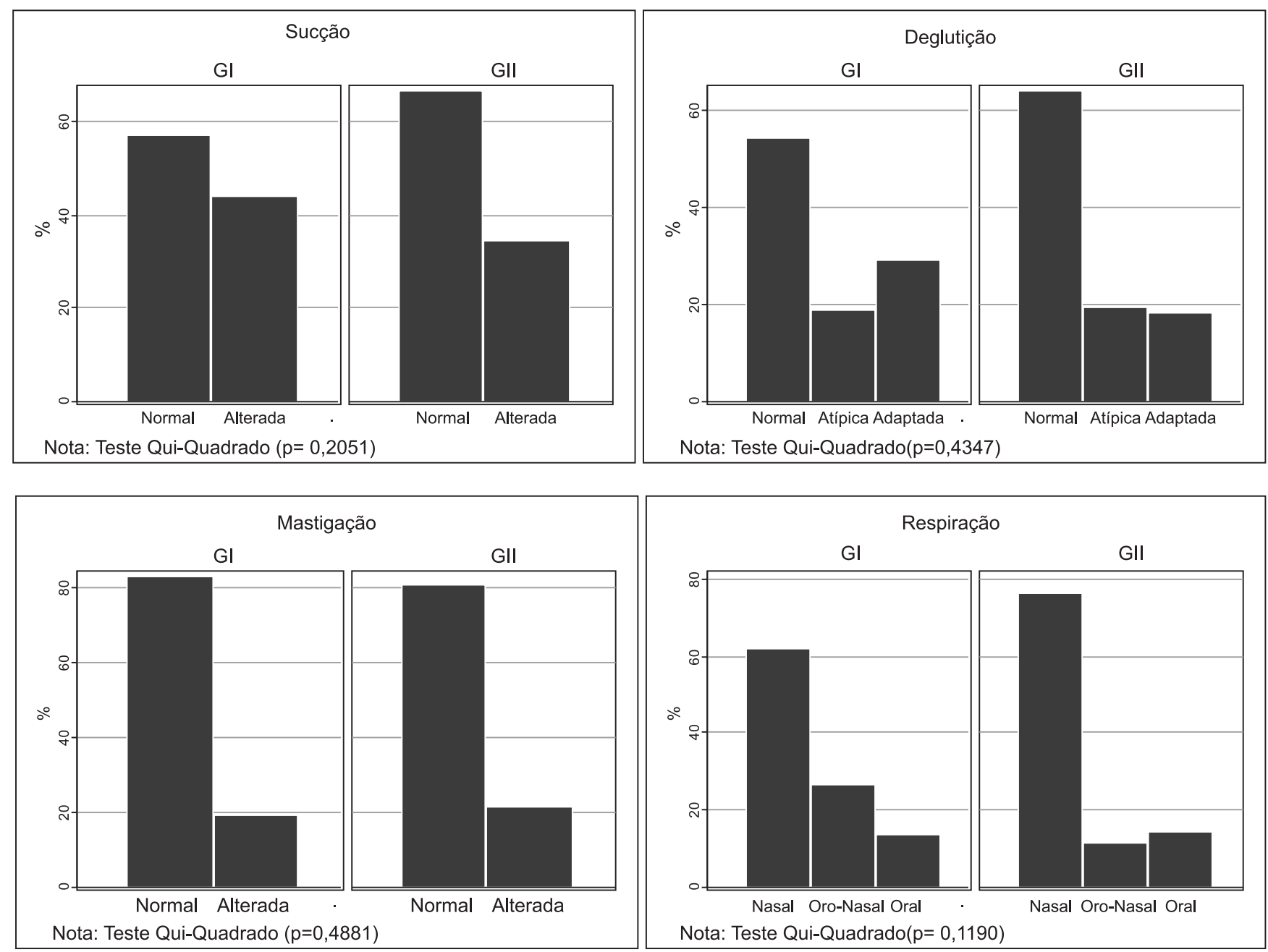

Legenda: $\mathrm{GI}$ = grupo I - fonema /r/ não estabelecido; GII = grupo II - fonema /r/ estabelecido

Figura 2. Comparação entre a aquisição do fonema /r/ e as funções do sistema estomatognático

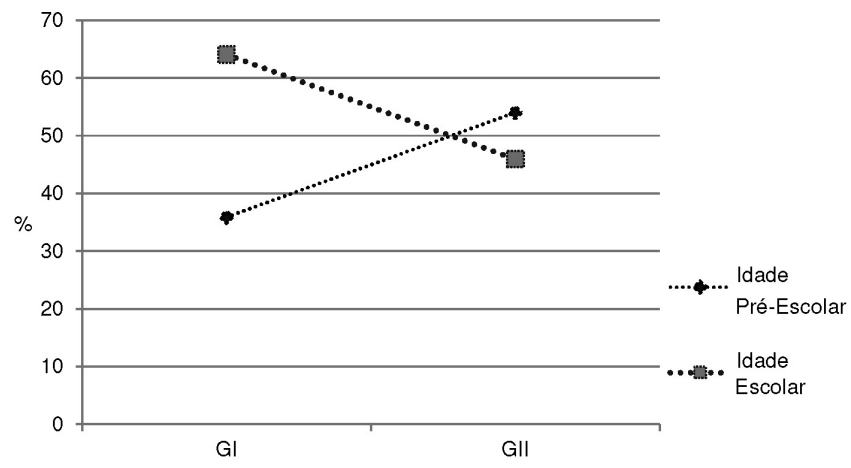

Legenda: GI = grupo I - fonema /r/ não estabelecido; GII = grupo II - fonema $/ \mathrm{r} /$ estabelecido

Figura 3. Aquisição do fonema /r/ segundo a idade

complexa a ser realizada. Um estudo ${ }^{(22)}$ refere que a praxia de vibrar a língua só é adquirida após a aquisição do /r/. Os achados desta pesquisa não são suficientes e específicos para comprovar tal afirmação, mas pode-se inferir que a praxia de vibrar a língua é uma habilidade difícil de ser realizada, em geral, pelas crianças com DF.

Esta pesquisa corrobora outro estudo ${ }^{(16)}$ que apresenta a pra-

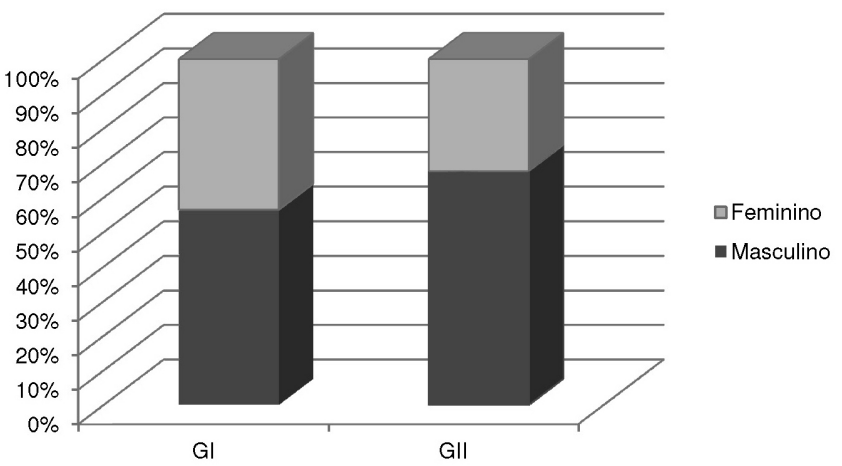

Legenda: $\mathrm{GI}$ = grupo I - fonema /r/ não estabelecido; GII = grupo II - fonema $/ r /$ estabelecido

Figura 4. Aquisição do fonema /r/ segundo o gênero

xia de vibração de língua como sendo a que mais diferenciou o grupo com o fonema /r/ estabelecido do grupo sem o /r/. Todas as crianças que apresentavam o fonema /r/ não estabelecido não realizavam vibração de língua. Ainda, este estudo concorda com outro ${ }^{(29)}$ que, de 12 crianças que apresentavam desvio de fala no fonema /r/, sete tiveram dificuldade para vibrar a língua.

Em relação às demais estruturas (lábios e bochechas) não 


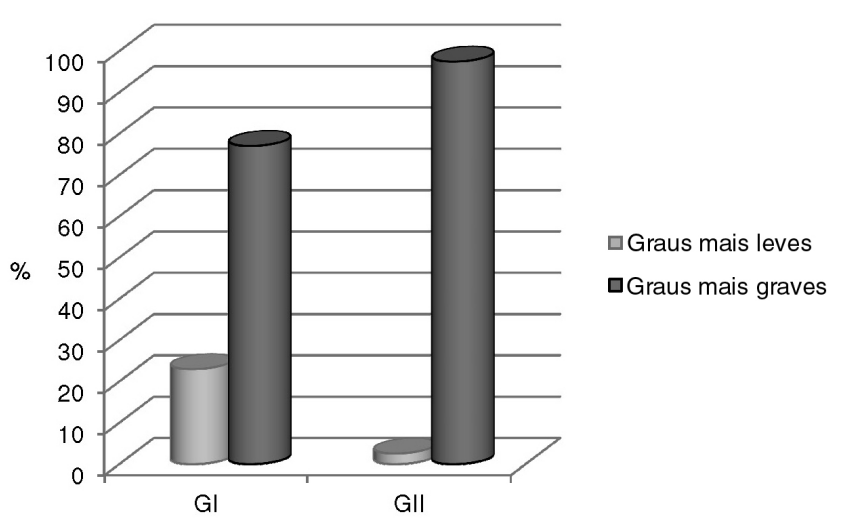

Legenda: $\mathrm{Gl}$ = grupo I - fonema /r/ não estabelecido; GII = grupo II - fonema $/ r /$ estabelecido

Figura 5. Aquisição do fonema /r/ conforme a gravidade do desvio fonológico

se observaram resultados que diferenciassem os grupos, o que evidencia que a língua é a principal estrutura do SEG envolvida na produção do fonema $/ \mathrm{r}^{(7)}$.

Um dos achados importantes a ser discutido diz respeito à oclusão. $\mathrm{O}$ fato de esta ser normal para a maioria das crianças, de ambos os grupos, sugere uma característica comum para o DF, diferindo do desvio fonético, no qual é frequente a presença de alteração na oclusão ${ }^{(10)}$. Isso foi confirmado nesta pesquisa, em que a análise dos resíduos ajustados revelou haver associação entre essas variáveis.

Neste estudo, a presença de alterações nas funções do SEG não mostrou relação com a aquisição do fonema $/ \mathrm{r} /$, sendo este resultado também encontrado em outro estudo ${ }^{(15)}$. O fato da presença de alterações nas funções do SEG não influenciar a produção do fonema /r/, pode ser justificado por se tratar de alterações mínimas, passíveis de adaptação para a produção do fonema $/ \mathrm{r} / \mathrm{e}$ de outros sons da fala ${ }^{(14,21)}$.

Um estudo ${ }^{(20)}$ analisou o sistema miofuncional de crianças com DF e relacionou com índices de estimulabilidade, esperando encontrar relação entre estes, pois a mesma é uma medida de produção de fala que auxilia no reconhecimento das dificuldades em nível fonético, fonológico ou de percepção ${ }^{(23)}$. As autoras observaram que 55,6\% dos sujeitos apresentaram alguma alteração no sistema miofuncional, o que não era o esperado.

De acordo com a literatura ${ }^{(9,11)}$, é comum encontrar alterações em uma ou mais das funções estomatognáticas em crianças com queixa de fala, o que concorda com os achados do presente estudo e de outras pesquisas ${ }^{(15,21)}$.
Os achados mostram que a idade é um fator considerável para o fonema /r/ estar estabelecido no SF das crianças com DF. Assim, com o aumento da idade, verifica-se também uma progressão na aquisição do fonema $/ \mathrm{r} /$. Esses achados concordam com a literatura ${ }^{(1,2)}$, a qual refere que em torno dos 5 anos de idade, em geral, as crianças já apresentam seu SF estabelecido.

A aquisição do fonema /r/ não sofre influência da variável gênero, embora seja afirmado que o DF é predominante no gênero masculino ${ }^{(10)}$.

No que se refere à gravidade do DF, os achados apontam que o fonema $/ \mathrm{r} /$ está frequentemente estabelecido no GL e não estabelecidos no GG. Tal achado sustenta a hipótese que o GL apresenta dificuldade principalmente nas estruturas silábicas mais complexas ${ }^{(30)}$, enquanto que no GG o comprometimento envolve as estruturas simples e complexas da aquisição do fonema $/ \mathrm{r} /$.

\section{CONCLUSÃO}

Os resultados encontrados, referentes às alterações nas funções, estruturas, tonicidade e mobilidade do SEG, permitem concluir que a aquisição do fonema /r/ não apresenta relação com tais alterações. Ainda, nas tarefas de afilar e alargar a língua, a maioria das crianças, independentemente de ter ou não o fonema $/ \mathrm{r} /$ estabelecido, apresentaram dificuldade em vibrar a língua. Aparentemente, não produzir o fonema /r/ não está diretamente relacionado à dificuldade práxica de vibrar a língua, o que permite concluir que a praxia de vibrar a língua é uma inabilidade inerente ao próprio DF.

No que se refere à oclusão, há predominância de oclusão normal em ambos os grupos, fato que permite inferir que a produção incorreta do /r/ não apresenta relação com a mal-oclusão. Além disso, reforça o conceito de o desvio fonológico envolver uma alteração no nível fonológico sem a presença de comprometimento orgânico que impeça a produção correta dos sons da fala.

Por sua vez, o fonema /r/ estar ou não estabelecido foi influenciado pela idade escolar. Isso se deve ao aumento de complexidade do sistema fonológico que emerge com o avanço da idade. Além disso, a aquisição do fonema /r/ não foi influenciada pela variável gênero.

Por fim, o fato da aquisição do fonema /r/ ser influenciada pela gravidade do desvio fonológico, permite concluir que crianças com desvios mais graves, em geral, apresentam maiores dificuldades na aquisição do fonema /r/, o que também repercute no estabelecimento da estrutura silábica com esse fonema. 


\begin{abstract}
Purpose: To compare the production of the phoneme /r/ in children with phonological disorder (PD) and the stomatognathic system (SS) alterations considering the variables age, gender, and severity of the PD. Methods: The sample was composed by 113 subjects with PD and SS alterations, of both genders, and with ages between 4 years and 8 years and 11 months, divided into two groups, considering the age range: pre-school age (4 years to 5 years and 11 months), and school age (6 years to 8 years and 11 months). The Child's Phonological Assessment and a stomatognathic system evaluation were carried out. The subjects were divided into two groups, according to the severity of PD: More Severe Degrees (MSD) and Milder Degrees (MD). Considering the phonological system, subjects were divided into GI: /r/ established, and GII: /r/ not established. The collected data were tabulated, analyzed and submitted to statistical analysis. Results: No differences were observed between GI and GII regarding tongue, lips and cheeks position, tonicity and mobility, as well as in SS structures and functions. GI obtained better performance only in tongue vibration. It was verified a correlation between the acquisition of phoneme /r/ and age increase; no correlation was found between the acquisition of /r/ and gender. MSD of PD were more frequent in GII, and MD in GI. Conclusion: There is no relation between alterations in the PS and in structures and functions of the SS in children with PD; PD is related only with children's age.
\end{abstract}

Keywords: Stomatognathic system; Speech; Child; Speech disorders; Articulation disorders

\section{REFERÊNCIAS}

1. Keske-Soares M, Donicht G, Checalin MA, Ghisleni MR. Generalização por 'reforço' ou 'contraste' no tratamento do desvio fonológico. Rev Soc Bras Fonoaudiol. 2008;13(4):391-7.

2. Newmeyer AJ, Grether S, Grasha C, White J, Akers R, Aylward C, et $a l$. Fine motor function and oral-motor imitation skills in preschool-age children with speech-sound disorders. Clin Ped. 2007;46(7):604-11.

3. Gierut JA. Treatment efficacy: functional phonological disorders in children. J Speech Lang Hear Res. 1998;41(1):S85-100.

4. Kent RD. Research on speech motor control and it disorders: a review and prospective. J Commun Disord. 2000;33(5):391-427.

5. Wohlert AB, Smith A. Development change in variability of lip muscle activity during speech. J Speech Lang Hear Res. 2002;45(6):1077-87.

6. Farias SR, Ávila CR, Vieira MM. Relação entre fala, tônus e praxia não-verbal do sistema estomatognático em pré-escolares. Pró-Fono. 2006;18(3):267-76.

7. Rigatti AP, Fonseca RP, Ramos AP. Aquisição normal e desviante do rótico alveolar simples em dois dialetos do português brasileiro. PróFono. 2001;13(2):157-64.

8. Shriberg LD. Diagnostic classification of five subtypes of childhood speech sound disorders (SSD) of currently unknown origin. In: Congress International Association of Logopedics and Phoniatrics (IALP); 2004 Aug 29-Sep 2; Brisbane, Australia. Proceedings.

9. Franco DP, Ávila CR. Achados fonoaudiológicos de crianças com queixa de distúrbio de fala. Pró-Fono. 2000;12(1):40-7.

10. Casarin MT, Gindri G, Keske-Soares M. Alterações do sistema estomatognático em distúrbios da fala. Rev Soc Bras Fonoaudiol. 2006;11(4):223-30.

11. Ahlsén E. Embodiment in communication - aphasia, apraxia and the possible role of mirroring and imitation. Clin Linguist Phon. 2008;22(45):311-5.

12. Green JR, Moore CA, Higashikawa M, Steeve RW. The physiologic development of speech motor control: lip and jaw coordination. J Speech Lang Hear Res. 2000;43(1):239-55.

13. Green JR, Moore CA, Reilly KJ. The sequential development of jaw and lip control for speech. J Speech Lang Hear Res. 2002;45(1):66-79.

14. Bleile K. Evaluating articulation and phonological disorders when the clock is running. Am J Speech Lang Pathol. 2002;11(3):243-9.

15. Morales RC. Terapia de regulação orofacial. São Paulo: Memnon; 1999. 195p.
16. Fonseca RP, Dornelles S, Ramos AP. Relação entre a produção do r-fraco e as praxias linguais na infância. Pró-Fono. 2003;15(3):229-40.

17. Bauman-Waengler J. Desenvolvimento fonológico normal. In: Lowe R. Fonologia - avaliação e intervenção: aplicações na patologia da fala. Porto Alegre: Artes Médicas; 1996. p. 33-65.

18. Pagan LO, Wertzner HF. Análise acústica das consoantes líquidas do Português Brasileiro em crianças com e sem transtorno fonológico, Rev Soc Bras Fonoaudiol. 2007;12(2):106-13.

19. Ramos AP. Aquisição dos róticos em um dialeto do português brasileiro: discussão de aspectos fonéticos e fonológicos e suas implicações para a clínica fonoaudiológica. Rev Soc Bras de Fonoaud. 2000;6(4):31-5.

20. Fraga L. Os róticos no português de Carambeí/PR. Estud Linguísticos. 2006;35:1113-22.

21. Castro MM, Wertzner HF. Estimulabilidade e tipo de erro de fala. Rev Soc Bras Fonoaudiol. 2006;11(1):1-9.

22. Monaretto VN. O status fonológico da vibrante. Letras de Hoje. 1994;29(1):153-7.

23. Wertzner HF, Sotelo MB, Amaro L. Analysis of distortions in children with and without phonological disorders. Clinics (São Paulo). 2005;60(2):93-102.

24. Glaspey AM, Stoel-Gammon C. Dynamic assessment in phonological disorders: the scaffoldind scale of stimulability. Top Lang Disord. 2005;25(3):220-30.

25. Yavas M, Hernandorena CL, Lamprecht RR. Avaliação fonológica da criança: reeducação e terapia. Porto Alegre: Artes Médicas; 1991.

26. Shriberg LD, Austin D, Lewis BA, McSweeny JL, Wilson DL. The speech disorders classification system (SDCS): extensions and lifespan reference data. J Speech Hear Res. 1997;40(4):723-40.

27. Bernhardt B. Developmental implications of nonlinear phonological theory. Clin Linguist Phon. 1992;6(4):259-81.

28. Marchesan IQ. Fundamentos em fonoaudiologia: aspectos clínicos da motricidade oral. 2a ed. Rio de Janeiro: Guanabara Koogan; 2005.

29. Gonçalves CS, Ferreira MC. Estudo da relação entre presença de frênulo lingual curto e/ou anteriorização e a dorsalização do fone /r/ na articulação da fala. Rev CEFAC. 2006;8(1):56-60.

30. Keske-Soares M. Terapia fonoaudiológica fundamentada na hierarquia implicacional dos traços distintivos aplicada em crianças com desvios fonológicos [tese]. Porto Alegre: Pontifica Universidade Católica do Rio Grande do Sul; 2001. 